\title{
Pteridines, Folic Acid Analogs
}

National Cancer Institute

\section{Source}

National Cancer Institute. Pteridines, Folic Acid Analogs. NCI Thesaurus. Code C1901.

Folic acid analogs containing a pteridine base, a two-ring heterocyclic aromatic

compound, and are used to interfere with folic acid. 